\title{
Advanced life support courses for dentists
}

\author{
Appropriateness of a Resuscitation Council (UK) advanced life support course for primary care dentists \\ P. Coulthard, C. M. Bridgman, A. Larkin, and H. V. Worthington Br Dent J 2000; 188: 507-512
}

\author{
Objectives \\ To investigate whether a standard Resuscitation Council (UK) \\ ALS course is appropriate for primary care dentists or whether a \\ course should be specifically designed for dentists.
}

\section{Design}

Opinions canvassed by pre-course expectation and post-course evaluation questionnaires.

\section{Subjects}

23 West Pennine primary care dentists providing a general anaesthetic or conscious sedation service who attended an ALS course.

\section{Results}

Knowledge and skills were rated on a 5-point scale from 1 (not important at all) to 5 (extremely important). Basic airway management $($ mean $=5)$ and anaphylaxis $($ mean $=4.9)$ scored the highest on the 'expectation' questionnaire. Rhythm recognition $(P<0.001)$, defibrillation $(P=0.007)$ and arrest algorithms $(P=0.047)$ were rated as significantly more important after the course than before. Knowledge about rhythm disorder management, cardiac pacing, post-resuscitation care, blood gas interpretation and bereavement were not considered to be so important either before or after the course.

\section{Conclusions}

Despite rating some aspects as unimportant, all dentists stated that this course had been appropriate. They did not want a specially designed ALS course for dentistry. Taking exactly the same recognised course and assessments as other healthcare professionals and gaining the same certification was felt to be important to this group of dentists.

\section{Comment}

7 he General Dental Council, in 'Main1 taining Standards' stresses the need for general dental practitioners to be prepared for a medical emergency by ensuring that all staff are adequately trained and can provide effective basic life support. The same level of preparedness applies whether conscious sedation is provided or not. Where general anaesthesia is provided, advanced life support (ALS) should be readily available in the case of a cardiac arrest.

The difficulty for practitioners is to know how far to go in preparing for the very rare serious medical emergency. Most practitioners will not see a serious medical emergency in their surgeries during their practising lifetimes.

This paper assesses the attitudes of several general dental practitioners toward acquiring the knowledge and skills necessary to provide advanced life support. All the practitioners in the survey were using sedation or working with anaesthetists providing general anaesthesia.

During the meeting before the course, attitudes were tested regarding the need for competency in a number of related skills. After the course, many of the candidates had changed their perception of the importance of many aspects of dealing with medical emergencies.

Subjects that were considered more important after the course included defibrillation, airway management and rhythm recognition, while opinions did not change regarding cardiac pacing, post-resuscitation care, blood gas interpretation and bereavement. Even these subjects, while considered unimportant were of interest to the candidates.

In the light of these findings, the candidates were asked whether a separate ALS course for dentists would be appropriate. It would deal merely with the subjects relevant to dental practice. This was rejected by those taking part as it would not have the same respect accorded to it. Nevertheless, the suggestion of a standard ALS course with only dentists as candidates was greeted enthusiastically as it was perceived as less threatening.

The ALS course did not include training in the handling of medical emergencies such as anaphylaxis, hypoglycaemia, epilepsy and angina. Candidates felt that such knowledge is important and should be dealt with on a separate course.

The paper is a useful contribution to the debate on training for emergencies and being prepared for the unexpected. Although there is no requirement for dentists to be ALS providers, most candidates felt that the skills acquired would stand them in good stead and were reassured that they were as ready as they could possibly be.

\section{Derek DeBuse}

Senior Demonstrator, Department of Sedation and Special Care Dentistry, GKT Dental Institute 